\section{La dottrina della giustificazione: uno sguardo ecumenico*}

\author{
Ashraf N. I. Abdelmalak ${ }^{* *}$
}

RECIBIDO: 20-03-15 - APROBADO: 06-07-15

Resumen: La questione della "giustificazione" è una questione attualissima che risponde a domande vitali e necessarie degli uomini di tutti i tempi ed, in particolare, degli uomini d'oggi: Che cos'è che rende il cristiano giusto davanti a Dio? L'essere giusti è frutto della sola nostra buona volontà? Qual è la salvezza che la fede cristiana promette? Il presente testo studia questa questione nell'importantissimo documento intitolato Dichiarazione congiunta sulla dottrina della giustificazione, per sottolineare le categorie di "consenso differenziato" e di "unità nella diversità riconciliata".

Palabras Clave: giustificazione, cattolici, luterani, ecumenismo, consenso differenziato.

PARA CITAR ESTE ARTÍCULO:

Abdelmalak, Ashraf. "La dottrina della giustificazione: uno sguardo ecumenico.” Theologica $\mathrm{Xa}$ veriana 180 (2015): 409-445. http://dx.doi.org/ 10.11144/javeriana.tx65-180.dgse

\section{La doctrina de la justificación. Una mirada ecuménica}

Resumen: La doctrina de la justificación es una cuestión de gran actualidad, toda vez que responde a preguntas vitales $y$ necesarias que se han planteado los hombres de todos los tiempos y, en particular, el hombre de hoy: ¿qué hace al hombre justo frente a Dios?, ¿Quién salva al hombre y le da plena realización a su vida? ¿Cuál es la salvación que la fe cristiana promete? El presente texto estudia esta cuestión en la llamada "Declaración conjunta sobre la doctrina de la justificación", para subrayar las categorías de "consenso diferenciado" y de "unidad en la diversidad reconciliada". Palabras Clave: Justificación, católicos, luteranos, ecumenismo, sentido diferenciado.

\section{The doctrine of justification. An ecumenical approach}

Abstract: The doctrine of justification is still a current issue, due to the fact it answers vital and necessary questions asked by men of all times and, particularly, by current men: What makes human being just in front of God? And, what is the salvation that Christian faith promises? The present text studies the issue of the "Joint Declaration on the Doctrine of Justification" with the aim to underline the dialogical categories "differentiated consensus" and "unity in the reconciled diversity".

KEY wORDS: Justification, Catholics, Lutherans, Ecumenism, Differentiated sense.

\footnotetext{
"Questo articolo è una riflessione elaborata a partire di una ricerca sviluppata all'interno di un seminario svolto nel ciclo dottorale all'Università Urbaniana a Roma.

** Dottore in Teologia dall'Università Urbaniana di Roma. Docente della Facoltà di Teologia della Universidad San Buenaventura (Bogotá). E-mail: ashrnaner3@hotmail.com
} 


\section{Introduzione}

Nonostante l'importanza della questione vitale della "giustificazione", la storia del cristianesimo dimostra che intorno ad essa c'erano delle tensioni tra i cristiani, e in modo particolare tra la Chiesa Cattolica romana e i Luterani. ${ }^{1}$ Perciò nel nostro presente lavoro abbiamo cercato prima di seguire lo sfondo storico delle condanne reciproche e della ricerca di nuovo ad una comprensione reciproca tra le due parti; e poi ci siamo soffermati a lungo sul contenuto dell'importantissimo documento intitolato Dichiarazione congiunta sulla dottrina della giustificazione, $D C D G^{2}$, perché con esso si è arrivato finalmente ad "un consenso tra luterani e cattolici su verità fondamentali di tale dottrina della giustificazione" (DCDG 40); e poi abbiamo voluto presentare la DCDG anche dal punto di vista ecumenico; e per ultimo, nella conclusione, abbiamo cerato di individuare una visione ecclesiologica dell'unità tra i cristiani che emerge da tale Dichiarazione.

Per quanto riguarda la struttura, quindi, abbiamo cercato di studiare la DCDG dal punto di vista storico, sistematico ed ecumenico. Infine, studiando il documento dedicato tutto alla questione della giustificazione, abbiamo tentato di delineare una visione ecumenica della Chiesa nascosta dentro le parole di tale importante documento.

\section{La Dichiarazione congiunta nella prospettiva storica}

Per parlare in modo corretto della DCDG, si deve ritornare alla storia del cristianesimo, ovvero al periodo che va dal Concilio di Trento fino ai nostri giorni, per capire meglio gli antecedenti di tale Dichiarazione. In effetti, per situarci bene nella prospettiva del risultato raggiunto è necessario ricordare che la dottrina della giustificazione fu nel

\footnotetext{
${ }^{1}$ A proposito della questione della giustificazione e della sua problematicità dal punto di vista biblico, storico e sistematico, c'è una letteratura ampia; si veda, ad esempio, Barth, Carta a los Romanos; 103-397; De Papiol, El protestantismo ante la Biblia, 75-81; Küng, La justificación. Doctrina de Karl Barth y una interpretación católica; Dettloff, "Justificación”, 480-490; Canals Vidal, En torno al diálogo católico-protestante, 30-48; Jeremias, Abbá y el mensaje central del Nuevo Testamento, 291-303; Eichholz, El Evangelio de Pablo. Esbozo de la teología paulina, 305-332; Wilckens, La Carta a los Romanos. Rom 6-16, II, 13-221; Ruiz de la Peña, El don de Dios. Antropología especial, 201-406; Ladaria, Teología del pecado original y de la gracia. Antropología teológica especial, 135-230; Jüngel, El Evangelio de la justificación del impio como centro de la fe cristiana. Estudio teológico en perspectiva ecuménica; Kasper, Cosechar los frutos. Aspectos básicos de la fe cristiana en el diálogo ecuménico, 55-72.

${ }^{2}$ Pontificio Consiglio per la Promozione dell'Unità dei Cristiani-Federazione Luterana Mondiale, "Dichiarazione congiunta sulla dottrina della giustificazione", 885-913. D'ora in poi sarà usata l'abbreviazione DCDG per rimandare a tale Dichiarazione.
} 
XVI secolo una questione nevralgica nella disputa tra Martino Lutero e le autorità della Chiesa Cattolica romana.

Le presentazioni tra loro contrastanti e la diversa comprensione di tale fondamentale dottrina, furono oggetto di condanne sia da parte del Concilio di Trento che delle confessioni luterane. Infatti, La DCDG osserva: "Gli scritti confessionali luterani e il Concilio di Trento della Chiesa cattolica emisero condanne dottrinali che sono valide ancora oggi e che hanno un effetto di separazione tra le Chiese" (DCDG 1). Questa importante osservazione rimanda consapevolmente alla storia, ossia alle condanne reciproche tra la Chiesa cattolica romana e i luterani. Perciò, cercheremo qui di presentare in modo riassuntivo questi antecedenti. Dopo aver fatto questo, daremo un breve sguardo sui documenti precedenti alla DCDG, i quali sono stati delle tappe importanti per arrivare allo stato di tale Dichiarazione.

\section{Martino Lutero e la questione della giustificazione}

La DCDG osserva:

La dottrina della giustificazione ha avuto un'importanza fondamentale per la Riforma luterana del XVI secolo. Essa l'ha considerata l'"articolo primo e fondamentale" e, al tempo stesso, la dottrina che "governa e giudica tutti gli altri aspetti della dottrina cristiana”. (DCDG 1).

Per tale motivo, continua la Dichiarazione:

...essa è stata particolarmente sostenuta e difesa, nella sua accezione riformata e nel suo valore particolare a fronte della teologia e della Chiesa cattolica romana del tempo, le quali sostenevano e difendevano da parte loro una giustificazione dagli accenti diversi. (DCDG 1).

Come è ben noto, la Riforma luterana in generale e la dottrina della giustificazione in particolare sono legate alla figura di Martino Lutero (1483-1546). Esso, infatti, è il padre della Riforma protestante, teologo ed esegeta. Nel 1515 ha preso a commentare la lettera ai Romani; e poi alla fine è venuto alla luce il suo famoso commentarius in Epistolom ad Romanos. Nel 1517, Lutero ha preso posizione contro l'autorità di Roma e del Papa. ${ }^{3}$ L’anno 1520 ha segnato la tappa conclusiva della Riforma luterana, perché

${ }^{3}$ La posizione di Lutero contro la Chiesa ed il Papa era "soprattutto contro l'abuso della predicazione delle indulgenze, indetta da Leone X per raccogliere i fondi necessari alla costruzione della basilica di S. Pietro a Roma, con la pubblicazione delle ben note Novantacinque tesi, che furono affisse alle porte della chiesa di Wittenberg" (Mondin, "Lutero Martin”, 357). 
in questo anno Lutero ha dato un volto definitivo e completo alla sua dottrina sulla giustificazione e ha proclamato pubblicamente la sua ribellione a Roma.

Tra gli scritti di Lutero ${ }^{4}$, troviamo la Confessio fidei augustana che nella Dieta di Augusto (1530) è riuscito a approvare. In realtà, in questo scritto si trova una sintesi della fede luterana, in cui si cerca di provare la conformità di questa fede con la Scrittura e con l'insegnamento della Chiesa anticha. In uno scritto del 1545, Lutero rievoca la sua esperienza di giovane monaco e la sua grande scoperta teologica. ${ }^{5}$ La giustizia di Dio è interpretata in senso passivo: è la giustizia che Dio dona, di cui egli investe l'essere del peccatore. Dio, nella sua misericordia, decide di fare grazia, di considerare giusto l'uomo, di perdonargli gratuitamente (per grazia) il peccato.

Lutero, infatti, ha scoperto che quando Paolo parlava della giustizia di Dio, non voleva affermare la giustizia che viene dalle nostre buone opere, ma quella che viene da Dio che ci accetta come peccatori. Lutero è arrivato a scoprire ed a sostenere che "non si tratta della nostra giustizia, ma della giustizia di Dio, giustizia che Dio ci dà per i meriti di Cristo, senza la nostra collaborazione, come sola grazia, e soltanto sulla base della fede (sola gratia, sola fides)" ${ }^{6}$

Certamente per comprendere bene la dottrina di Lutero in generale e la sua dottrina sulla giustificazione in particolare, si devono rendere presenti alcune nozioni fondamentali nella sua dottrina: la sola fides, la sola Scriptrura, la theologia crucis, la doppia predestinazione, etc. ${ }^{7}$ Inoltre, la dottrina di Lutero sulla giustificazione è stata condannata dalla Chiesa Cattolica ${ }^{8}$, in particolare nel Concilio di Trento. E questo sarà il nostro punto successivo.

${ }^{4}$ Tra le opere di M. Lutero si trovano anche il Piccolo catechismo (1528) e il Grande catechismo (1529) che con la Confessio fidei augustana sono le principali fonti per la dottrina della giustificazione. Cfr. Ibid., 357-358.

${ }^{5}$ Con la famosa "esperienza della torre" (1513) è venuta una profonda "conversione" intellettuale e spirituale in Lutero. In questa circostanza, infatti, con la lettura di (Rom 1,17), Lutero ha avuto una illuminazione sulla natura della giustificazione. Cfr. Ibid. 361.

${ }^{6}$ Kasper, "La Dichiarazione congiunta sulla dottrina della giustificazione. Un motivo di speranza", 8-9.

${ }^{7}$ Nella teologia di Lutero, ci sono due principi fondamentali: un principio architettonico (ovvero un contenuto) e un principio ermeneutico (ovvero una forma). Il principio architettonico è svolto dalla dottrina della giustificazione; mentre il principio ermeneutico è svolto dalla sola fides o sola Scriptura. Cfr. Mondin, "Lutero Martin", 358-370.

${ }^{8}$ In occasione della sua visita pastorale in Germania (21-23 giugno 1996), durante l'incontro coi rappresentanti delle chiese evangeliche e col gruppo di lavoro delle chiese cristiane in Germania (Sabato 22 giugno), Giovanni Paolo II afferma: "Dopo secoli di dolorosa estraneità e di discussione, il suo ricordo [di M. Lutero] ci permette oggi di riconoscere più chiaramente l'alta importanza della sua richiesta di una teologia vicina alle Sacre Scritture e della sua volontà di un rinnovamento spirituale della Chiesa. 


\section{Il Concilio di Trento e la dottrina cattolica della giustificazione}

Trattando di diverse questioni che riguardano la dottrina cattolica, il Concilio di Trento, nel suo Decreto sulla giustificazione nella sua sesta sessione del 13 gennaio del 1547, affronta tanti elementiriguardanti la questione della giustificazione. ${ }^{9}$ Esso non nasconde i danni avvenuti a causa di una dottrina erronea sulla giustificazione; e osserva che,

...in questi anni è stata divulgata con grave danno per molte anime e per l'unità della Chiesa, una dottrina erronea sulla giustificazione. Perciò questo sacrosanto Concilio Tridentino ecumenico e generale, legittimamente convocato nello Spirito Santo, a lode e gloria di Dio onnipotente, per la tranquillità della Chiesa e per la salvezza delle anime [...] intende esporre a tutti i fedeli cristiani la vera e sana dottrina sulla giustificazione... ${ }^{10}$

Dopo aver esposto con chiarezza e fermezza la dottrina cattolica sulla giustificazione, affinché i credenti sappiano ciò che devono credere e seguire, il Concilio di Trento ha aggiunto 33 canoni in cui ha elencato ciò i credenti debbano evitare e fuggire. In sostanza, i 33 canoni contengono condanne a dottrine errate sulla giustificazione. In fatti, in questi canoni la forma ripetuta è "se qualcuno afferma che [...] sia anatema". ${ }^{11}$ Per avere una sintesi breve e precisa sul contenuto della dottrina cattolica sulla giustificazione come è stata esposta nel concilio tridentino, troviamo nelle parole seguenti un gran aiuto:

Il Concilio di Trento [osserva W. Kasper] non fu in grado di accettare questa dottrina [quella di Lutero], così come essa era compresa a quel tempo. Certamente, anche Trento ha condannato la dottrina pelagiana secondo la quale una persona può redimere se stessa attraverso le buone opere. Tuttavia, il Concilio concluse che noi possiamo cooperare alla nostra giustificazione, non con la nostra propria forza, ma perché la grazia ci ispira e ci abilita a farlo. Inoltre il Concilio voleva

\footnotetext{
Il suo straordinario contributo allo sviluppo della lingua tedesca e la sua eredità culturale sono fuori discussione. La sua attenzione per la Parola di Dio e la sua risolutezza a percorrere il cammino di fede riconosciuto come giusto, non fanno tuttavia certamente ignorare i suoi limiti personali e altrettanto poco il fatto che i problemi fondamentali nel rapporto fra fede, Scrittura e tradizione e Chiesa, così come li ha visti Lutero, fino ad oggi non sono stati ancora sufficientemente chiariti." (Giovanni Paolo II, "Un ecumenismo promettente per il futuro può esistere solo ponendosi disinteressatamente di fronte alla verità", 1561-1567.

${ }^{9}$ Concilio di Trento, “Decreto sulla giustificazione (1547)", 647-671. D’ora in poi sarà usata l'abbreviazione $H D$ per rimandare a tale documento.

${ }^{10}$ Ibid., n. 1520.

${ }^{11}$ Ibid., nn. 1551-1583.
} 
mettere in chiaro che Dio non soltanto ci ha dichiarati giusti, ma ci ha resi giusti; che ci ha santificati e, senza meriti da parte nostra, ci ha rinnovati, in modo che, per mezzo della grazia -come affermano le Sacre Scritture-, noi siamo una nuova creazione. Conseguentemente, noi dobbiamo vivere come "una creatura nuova". La fede deve diventare effettiva nell'amore e nelle azioni di carità. ${ }^{12}$

Il Concilio di Trento termina il suo Decreto sulla giustificazione nel canone 33, con queste parole:

Se qualcuno afferma che con questa dottrina cattolica della giustificazione, espressa dal santo sinodo col presente decreto, si riduce in qualche modo la gloria di Dio o i meriti di Gesù Cristo nostro Signore, e non si manifesta piuttosto la verità della nostra fede e infine la gloria di Dio e di Gesù Cristo: sia anatema. ${ }^{13}$

Ciò vuol dire, al di là del linguaggio che a noi oggi sembra duro, che il concilio ha ribadito la dottrina della Chiesa cattolica a proposito della giustificazione, riaffermando la continuità con la tradizione apostolica che è stata trasmessa a noi sotto la guida dello Spirito Santo.

\section{Il cammino verso la Dichiarazione congiunta}

A proposito della DCDG, il cardinale Kasper dice che tale documento è stato realizzato grazie ad un lungo e difficile processo di intenso dialogo svolto sotto gli auspici del Pontificio Consiglio per la Promozione dell'Unità dei Cristiani (PCPUC) e della Federazione Luterana Mondiale (FLM) ${ }^{14} \mathrm{Al}$ di là di ogni dubbio, esso deve essere inteso come un eminente risultato del movimento ecumenico ed una pietra miliare lungo il cammino verso il ristabilimento della piena unità visibile tra i discepoli dell'unico signore e salvatore Gesù Cristo. ${ }^{15}$

In realtà, per la tradizione luterana e le chiese luterani, la dottrina della giustificazione ha conservato un particolare valore. "Per questo motivo essa ha assunto fin dall'inizio un posto importante anche nel dialogo ufficiale luterano-cattolico"

\footnotetext{
${ }^{12}$ Kasper, "La Dichiarazione congiunta sulla dottrina della giustificazione", 9.

${ }^{13}$ Concilio di Trento, "Decreto sulla giustificazione”, 1551-1583.

${ }^{14}$ La Federazione Mondiale Luterana (Lutheran World Federation, LWF) è un'alleanza di chiese nazionali e regionali che aderiscono alla confessione luterana fondata nel 1947. Ha sede a Ginevra. Forte della partecipazione di 140 chiese, rappresentanti 78 paesi, raccoglie 66 milioni di protestanti luterani. Membro italiano è la Chiesa Evangelica Luterana in Italia.
}

${ }^{15}$ Kasper, "La Dichiarazione congiunta sulla dottrina della giustificazione", 10. 
(DCDG 2). Qui, si pensa certamente alle varie fasi del dialogo internazionale luteranocattolico, ovvero ad alcuni importanti rapporti precedenti, come ci rimanda la stessa Dichiarazione (DCDG n. 3).

Ora, vorremmo percorre in modo riassuntivo tale lungo processo, limitandoci ai documenti che riguardano in modo particolare il nostro tema; e ciò ci aiuterà $a$ rendere conto del cammino fatto, e quindi dell'importanza dell' accordo raggiunto tra la Chiesa Cattolica e i Luterani. Percorrendo il camino fatto, a partire dal 1972 fino al 1999, daremo solamente un breve sguardo storico e dottrinale su ciascun documento.

\section{"Il Vangelo e la Chiesa" (1972)}

Questo primo documento Il Vangelo e la Chiesa ${ }^{16}$, chiamato anche Rapporto di Malta, è frutto di una lunga e faticosa preparazione che ha avuto origine nel lavoro stesso del Concilio Vaticano II. ${ }^{17}$ Infatti, i contatti tra la FLM e la Chiesa Cattolica romana, iniziati con la partecipazione di alcuni osservatori luterani a tale Concilio, hanno portato alla costituzione di un gruppo di lavoro composto da cattolici e luterani. Questo gruppo si è unito due volte a Strasburgo nel 1965 e nel 1966. Esso doveva occuparsi di due dialoghi: Il Vangelo e la Chiesa e La teologia del matrimonio e il problema dei matrimoni misti.

Riguardante il primo dialogo, si comincia già nel 1967, con la costituzione di una commissione di studio luterana/cattolica, composta di 14 membri. Questa commissione, dopo cinque incontri dal 1968 fino al 1971, ha prodotto un rapporto su $I l$ Vangelo e la chiesa (Rapporto di Malta). Dopo la pubblicazione di tale documento, si è formata la commissione congiunta cattolica/luterana. Questa commissione, come anche la precedente, sono designati dal comitato esecutivo della FLM e dal Segretariato per l'unione dei Cristiani. E così si procede nel dialogo teologico su altre questioni che non erano discusse sufficientemente nel Rapporto di Malta.

\footnotetext{
${ }^{16}$ Per tutto ciò che viene detto qui a proposito di tale documento, si veda Cereti e Voicu (cur.), Enchiridion oecumenicum 1,1129 ss.

${ }^{17}$ Ai cardinali e ai collaboratori della Curia Romana nel corso della tradizione incontro nella Basilica Vaticana (venerdì 28 giugno 1985), il papa Giovanni Paolo II dice: "Infatti, il 5 giugno 1960, allora giorno di Pentecoste, Giovanni XXIII istituiva, con il Motu Proprio 'Superno Dei Nutu', le undici commissioni che avrebbero preparato il Concilio e, insieme con esse, un Segretariato per la promozione dell'unità cristiana. Il Papa aveva trovato così il mezzo per assicurare, nella preparazione del Concilio e durante il suo svolgimento, la presenza permanente della sollecitudine di restaurare l'unità cristiana; di associare a questa preoccupazione e a questo impegno, i rappresentanti delle altre Chiese e comunità ecclesiali e di fare in modo che il Concilio diventasse, in una certa misura, un avvenimento 'a edificazione e letizia di tutto il popolo cristiano'." (Giovanni Paolo II, "L'impegno della Chiesa nell'ecumenismo deriva da una decisione irrevocabile", 1988).
} 
A proposito, invece, dello scopo e del contenuto di tale documento, si possono essere compresi dal titolo stesso del documento Il Vangelo e la chiesa. In realtà, il lavoro svolto aveva lo scopo di sottoporre a una nuova analisi le differenze confessionali, alla luce delle più recenti acquisizioni bibliche, teologiche e storiche, e sulla base delle nuovo prospettive aperte dal Concilio Vaticano II, come dice il documento stesso.

Partendo dal tema principale, ovvero il legame tra il Vangelo e la Chiesa, il documento sviluppa altri importanti temi. Essotratta, per primo, il legame tra il Vangelo e la tradizione, e afferma che non si può contrapporre la Scrittura alla tradizione come se vi fosse estranea, perché lo stesso NT è il risultato della tradizione cristiana primitiva; anche se la Scrittura ha una finzione normativa.

E poi il documento ha trattato della questione del Vangelo e il Mondo; e a proposito di questo, la cosa più importante da dire è che il Vangelo si dischiude per noi nel suo significato salvifico solamente se viene annunciato nel contesto di situazioni concrete. Un'altra questione legata al Vangelo è quella del ministero nella Chiesa. Partendo dal Vangelo, si è cercato una nuova interpretazione della dottrina tradizionale del ministero. Infine, il documento ha trattato il tema del Vangelo e l'unità della Chiesa. In tale contesto, certamente, si è visto la questione del primato del Papa.

Si può affermare che il rapporto su Il Vangelo e la chiesa parla fin dall'inizio di un "ampio consenso" sulla questione della giustificazione; ciò vuole significare che fin dai loro primi incontri, nel 1967, i partecipanti al dialogo internazionale avevano constatato tale "ampio consenso". A dire il vero, questo documento che riassume i risultati del lavoro svolto rivela la profonda comunione di fede che esiste tra le due chiese ed indica anche le questioni che richiedono uno studio ulteriore.

\section{“Giustificazione per fede” (1983)}

Questo documento Giustificazione per fede $e^{18}$ è uno dei frutti di un lungo dialogo bilaterale tra cattolici e luterani; esso è frutto di sei anni di lavoro della commissione composta da alcuni studiosi nominati rispettivamente dal Comitato Episcopale cattolico degli Stati Uniti per gli affari ecumenici e interreligiosi e dai Ministeri luterani mondiali. Il dialogo svolto è iniziato dal 1965, e ha discusso diversi importanti temi.

In realtà, grazie a questa commissione i luterani e cattolici degli Stati Uniti hanno svolto un dialogo teologico riguarda alle dottrine che hanno unito o separato l'una dall'atra le loro chiese a partire dal XVI secolo. Sebbene l'argomento della

${ }^{18}$ Per tutto ciò che viene detto qui a proposito di tale documento, si veda Cereti (cur.), Enchiridion oecumenicum 2, 1129 ss. 
"giustificazione per la fede" sia stato toccato in precedenti sessioni del dialogo, è solo in questo documento del 1983 che esso è stato esaminato a fondo. La Dichiarazione si basa sulle discussioni, iniziate nel 1978, di relazione riassuntive di una notevole mole di ricerche bibliche, storiche, teologiche ed ecumeniche.

Se vogliamo, invece, presentare brevemente il contenuto dottrinale di tale documento, dobbiamo iniziare dicendo che questo documento è il risultato di un processo di ricerca comune, e non un compromesso tra opinioni inizialmente in contrasto. La Dichiarazione ha cercato di mostrare come si sono sviluppate le discordanze storiche nell'interpretazione della dottrina biblica della giustificazione e in quale misura possono essere oggi superate.

Il documento, quindi, ha trattato per primo la storia del problema, partendo dal periodo prima del XVI secolo, poi nel XVI secolo, e infine dopo il XVI secolo. Una seconda tappa del documento è quella della riflessione e della interpretazione del termine "giustificazione" e di altri temi legati alla questione (come la peccaminosità del giustificato, la sufficienza della fede ...etc.).

Infine, si è cercato di partire dai dati biblici a proposito di tale questione (le Scritture ebraiche, la letteratura paolina, gli altri scritti del NT), per offrire delle prospettive e ricostruzioni. Non ci prolunghiamo qui a parlare dettagliatamente della dottrina della giustificazione che emerge da questo documento, perché avremo in seguito la possibilità di approfondirla con il documento della DCDG.

\section{"Le condanne dottrinali dividono ancora le Chiese?" (1986) ${ }^{19}$}

I membri della Commissione Ecumenica Congiunta nel 1980 hanno proposto di trattare, in modo storico e sistematico nel dialogo ecumenico, le condanne dottrinali del XVI secolo da parte della Chiesa Cattolica e delle Dichiarazioni confessionali. Ma tale proposta è stata seguita solamente nel 1986. L'approfondimento teologico del consenso sulla dottrina della giustificazione è stato compiuto da due gruppi di studio nazionali.

Il primo è stato il dialogo nazionale luterano-cattolico degli Stati Uniti, che ha presentato le proprie conclusioni nel 1983, come abbiamo visto sopra. Il secondo è stato il dialogo della Commissione tedesca, formata dopo la visita di Giovanni Paolo II in Germania (1985), con il compito di verificare la pertinenza delle condanne dottrinali ereditate dalla storia. Il tema della giustificazione, infatti, è stata affrontata

${ }^{19}$ Per tutto ciò che viene detto qui a proposito di tale documento, si veda anche Cereti (cur.), Enchiridion oecumenicum 2, 1129 ss. 
dopo tale visita del Papa Giovanni Paolo II in Germania, nell'ambito di uno studio che riguardava tutte le condanne dottrinali del XVI secolo. Gli esiti del Dialogo cattolico-luterano a livello tedesco erano pubblicati nel 1986 in un libro con il titolo Le condanne dottrinali dividono ancora le Chiese?, nel quale si giungeva ancora alla conclusione che oggi queste questioni non dividono più le chiese.

Grazie a questo studio molte controversie del XVI secolo appaiono oggi sotto una nuova luce. Questo progresso è stato possibile perché si è prestata, dal lato metodologico, un'accurata attenzione alla distinzione tra il contenuto della fede e la formulazione in cui esso viene espresso. ${ }^{20}$ Tale distinzione è, in effetti, uno degli elementi importanti per l'intesa ecumenica. Come insegna il Concilio Vaticano II: "Per ristabilire o conservare la comunione e l'unità bisogna "non imporre altro peso fuorché le cose necessarie (At 15,28)". ${ }^{21}$

A proposito di tale documento, sono anche molto significative le parole seguenti del papa Giovanni Paolo II:

Molti sinodali si sono seriamente confrontati con i risultati di questo documento di studio [Le condanne dottrinali sono tale da dividere la chiesa?]. Sono grato per tutti i chiarimenti ottenuti grazie ai sinodi. Prendo anche atto del fatto che espressioni polemiche e sconvenienti del passato sono state ritrattate e rimesse all'oblio storico. ${ }^{22}$

\section{"Chiesa e giustificazione" (1994)}

Il dialogo tra cattolici e luterani, come abbiamo già visto, ha avuto dei fasi nello svolgimento del suo lavoro. La prima fase del dialogo è cominciatanel 1967 a Zurigo concludendosi con il Rapporto di Malta o Il Vangelo e la chiesa, nel 1972. La seconda fase del dialogo è iniziata dal 1973 fino al 1984. In questa fase del lavoro ecumenico è uscita una serie di documenti che studiavano problemi dottrinali specifici. La terza fase,

\footnotetext{
${ }^{20}$ Giovanni Paolo II, “Lettera enciclica Ut unum sint sull'impegno ecumenico (1995)” 81, Vatican, http:// w2.vatican.va/content/john-paul-ii/it/encyclicals/documents/hf_jp-ii_enc_25051995_ut-unum-sint. html (consultato 20 di aprile 2015).

${ }^{21}$ Concilio Vaticano II, “Decreto sull'ecumenismo Unitatis redintegratio (1964)” 18, Vatican, http:// www.vatican.va/archive/hist_councils/ii_vatican_council/documents/vat-ii_decree_19641121_unitatisredintegratio_it.html (consultato 20 di aprile 2015).

${ }^{22}$ Giovanni Paolo II, "Un ecumenismo promettente per il futuro può esistere solo ponendosi disinteressatamente di fronte alla verità", 1561-1567.
} 
infine, ha iniziato il suo lavoro nel 1986 e l'ha concluso nel 1993 con l'approvazione del documento ufficiale Chiesa e giustificazione. ${ }^{23}$

Il documento che è stato pubblicato alla conclusione della terza fase del dialogo tra la Chiesa cattolica e la Comunione Luterana ha l'obiettivo, come anche del resto il dialogo stesso tra cattolici e luterani, di raggiungere l'unità visibile della Chiesa. Il documento, infatti, ribadisce che esiste un "largo consenso dottrinale", e esso invita a seguire passi concreti verso la piena comunione.

Il documento ha come chiave il significato della Chiesa alla luce della sacramentalità e della giustificazione dal momento che la giustificazione proviene dall'annuncio e dall'accoglienza del Vangelo. Nei primi capitoli del documento si parla su ciò che i cattolici e luterani condividono insieme sulla Chiesa e sulla giustificazione. E poi viene un capitolo sulla Chiesa quale recettrice e mediatrice di salvezza; e questo capitolo costituisce il cuore del documento, perché in esso si sono trattati i punti controversi sulla Chiesa (Chiesa sacramento della salvezza, carattere visibile e nascosto della Chiesa, etc.). Infine, il tema principale dell'ultimo capitolo è quello della missione e del compimento della Chiesa.

\section{"Dichiarazione congiunta sulla dottrina della giustificazione" (1999)}

Riassumendo le tappe essenziali che abbiamo percorso fino ora, si può osservare che prima del Concilio Vaticano II c'erano solo dei colloqui informali; e che il dialogo ufficiale, invece, inizia dopo il Concilio, nel 1967. Il comune ascolto della sacra Scrittura e l'accettazione delle conoscenze della scienza biblica, gli studi teologici e storici, sono la causa di un avvicinamento nella comprensione della giustificazione.

Nel 1972 con Il Vangelo e la Chiesa si profila la possibilità di una interpretazione concorde della giustificazione. Ma il contributo decisivo lo hanno dato i seguenti documenti: Giustificazione e fede (Usa, nel 1985), Le condanne dottrinale sono tale da dividere la chiesa?(Germania, nel 1986) e Chiesa e giustificazione (Germania, nel 1994).

Si può quindi dire che i lavori e le conclusioni dei due gruppi (quello statunitense e quello tedesco) hanno avuto un'ampia eco e suscitato dibattiti contrastanti in seno alle chiese. Il dialogo statunitense e tedesco ha confermato i significativi avvicinamenti alla comprensione della giustificazione, a tal punto che i partecipanti al dialogo tedesco hanno potuto proporre alle chiese di dichiarare che gli anatemi del XVI secolo non riguardano più l'insegnamento attuale delle Chiese.

\footnotetext{
${ }^{23}$ Per tutto ciò che viene detto qui a proposito di tale documento, si veda Cereti e Puglisi (cur.), Enchiridion oecumenicum 3, 1129ss.
} 
Nel 1994 il PCPUC e la FLM hanno incaricato un gruppo di preparare un testo conciso che riassumesse i trent'anni di lavoro. Nel 1997 si giungeva alla stesura finale della DCDG, che era sottoposta per esame alle autorità di tutte e due parti in vista della loro approvazione, cioè ai sinodi delle varie chiese luterane e, per la Chiesa cattolica, alla Congregazione per la Dottrina della Fede ed al PCPUC.

La FLM ha dato la sua risposta ufficiale il 16 giugno 1998 (la Risoluzione) e Roma ha reso nota la sua risposta il 25 giugno 1998 (la Risposta). Le due risposte hanno accolto in modo favorevole la DCDG, rilevando al tempo stesso che ci sono diverse questioni aperte e hanno bisogno di un complemento di dialogo. Dopo un altro anno di lavoro, nella primavera del 1999 un breve Allegato esplicativo ha permesso di risolvere le ultime difficoltà riguardanti le condanne reciproche. Al riguardo, partendo dal consenso raggiunto su verità fondamentali della giustificazione nella DCDG e dalle affermazioni stesse della DCDG, l'Allegato osserva: "Risulta pertanto chiaro che le reciproche condanne dei tempi passati non si applicano alla dottrina cattolica e alla dottrina luterana della giustificazione così come tali dottrine sono presentate nella Dichiarazione congiunta." ${ }^{24}$

Quest'importante espressione viene ripresa in posizione centrale anche dalla Dichiarazione ufficiale comune. Il 31 ottobre 1999 ad Augsburg (Augusta), nella chiesa luterana di Sant'Anna, era un grande avvenimento di pace e di riconciliazione, ovvero il giorno dell'atto formale della firma della Dichiarazione ufficiale comune con cui le autorità cattoliche e luterane hanno ratificato la DCDG.

In questo grande giorno è stato raggiunto un consenso tra cattolici e luterani sulla dottrina della giustificazione. In realtà, la DCDG ha una doppia finalità: Affermare l'accordo su "verità fondamentali della dottrina della giustificazione" e sottolineare che le reciproche condanne dottrinali del XVI secolo non colpiscono più l'insegnamento delle due parti in dialogo così come esso è presentato nella DCDG.

È veramente un momento importante nella storia della cristianità occidentale. In realtà, dopo 450 anni di separazione e condanne reciproche, come si è visto, cattolici e luterani sono arrivati a un consenso teologico sul modo di comprendere la salvezza operata da Gesù Cristo. Per tutto questo tempo la dottrina della giustificazione ci ha divisi come cattolici e luterani. ${ }^{25} \mathrm{E}$ la cosa da osservare qui è che le tragedie dell'umanità

\footnotetext{
${ }^{24}$ Cereti e Puglisi (cur.), Enchiridion oecumenicum 7, 1887ss.

25 "Alle soglie del terzo millennio la Federazione Luterana Mondiale e la Chiesa Cattolica affermano in modo vincolante che le chiese separate fanno affermazioni comuni sulla dottrina della giustificazione,
} 
in generale e del nostro tempo in particolare, hanno spinto i cristiani a guardare alla loro unità con uno sguardo nuovo. In realtà,

...soltanto nel loro comune rifiuto del sistema inumano del nazismo, nei bunkers della seconda guerra mondiale, e nei campi di concentramento, molti cristiani cattolici ed evangelici hanno compreso di non essere tanto lontano tra loro come poteva all'apparenza sembrare. Essi hanno compreso che ciò che li univa era più grande di ciò che li divideva. ${ }^{26}$

\section{La Dichiarazione congiunta nella prospettiva sistematica}

Arrivati a questo punto della nostra ricerca, vorremmo ora esaminare da vicino il contenuto della DCDG. Ciò ci aiuterà a comprendere il modo nuovo di interpretare la dottrina della giustificazione nella prospettiva ecumenica. In questa esposizione cercheremo di seguire il contenuto della DCDG facendo delle sintesi e delle valutazioni su tale contenuto.

\section{Messaggio biblico della giustificazione}

Dopo aver parlato dell'importanza fondamentale della dottrina della giustificazione per la Riforma Luterana del XVI secolo, la DCDG rimanda ai rapporti precedenti ad essa (DCDG nn. 1-7). ${ }^{27}$ E poi la DCDG precisa:

Questa Dichiarazione non contiene tutto ciò che si insegna in ciascuna Chiesa sulla giustificazione; tuttavia essa esprime un consenso su verità fondamentali della dottrina della giustificazione, mostrando come elaborazioni che permangono diverse non sono più suscettibili di provocare condanne dottrinali. (DCDG n. 5).

A tale punto, la DCDG richiama il messaggio biblico riguarda la dottrina della giustificazione (nn. 8-12). In realtà, grazie all'ascolto della Parola di Dio nella Sacra Scrittura le due parti si sono condotti ad alcune valutazioni nuove. I cattolici e luterani

che è stata nel XVI secolo l'origine e, in ultima analisi, il vero motivo della rottura dell'unità della chiesa d'Occidente. Da ciò consegue che anche le condanne dottrinali che si riferiscono alla dottrina della giustificazione non sono più tali da dividere le chiese." (Lehmann, "Fondamentalmente uniti", 708).

${ }^{26}$ Kasper, "La Dichiarazione congiunta sulla dottrina della giustificazione", 9.

${ }^{27}$ I documenti a cui rimanda la DCDG sono i seguenti: Il Vangelo e la Chiesa (1972), Giustificazione per fede (1983) e Lehrverurteilungen - kirchentrennend? (Le condanne dottrinali dividono ancora le Chiese?) [1986], Chiesa e giustificazione (1994). A proposito, cfr. DCDG 3. 
hanno riscoperto che la Sacra Scrittura offre la buona novella; e essa, a sua volta, viene rappresentata in diversi modi. Ma tra queste descrizioni della buona novella, la "giustificazione" del peccatore nella fede per mezzo della grazia di Dio ( $\mathrm{Rm} 3,23-25)$ ha un posto di spicco e speciale.

La giustificazione stessa viene descritta in diverse maniere. Essa, infatti, è perdono dei peccati, liberazione dal potere di dominio esercitato dal peccato e dalla morte e liberazione dalla maledizione della Legge. Essa è già da ora un essere accolti nella comunione con Dio, ma lo sarà pienamente nel regno di Dio che viene. La giustificazione unisce a Cristo, alla sua morte e risurrezione. Essa si realizza nel ricevere lo Spirito Santo nel battesimo il quale è incorporazione nell'unico corpo. Tutto questo viene unicamente da Dio, a causa di Cristo, per opera della grazia mediante la fede.

I giustificati vivono della fede che sgorga dalla parola di Cristo e agisce nell'amore, il quale è frutto dello Spirito. A causa delle concupiscenze, i giustificati debbono sempre di più porsi all'ascolto delle promesse di Dio, confessare i loro peccati, partecipare al corpo e al sangue di Cristo ed essere esortati a vivere in modo conforme alla volontà di Dio e in modo giusto (cfr. DCDG, nn. 8-12).

\section{La giustificazione come problema ecumenico}

La DCDG nel (n. 13) ha spiegato che le interpretazioni contraddittorie del messaggio biblico della giustificazione sono state nel XVI secolo una causa primaria della divisione della Chiesa d'Occidente, e che si è spesso anche avuto effetti sulle condanne dottrinali. Da qui la DCDG dice che una comune comprensione della giustificazione è quindi fondamentale e indispensabile per il superamento della divisione delle Chiese.

In realtà, grazie agli studi biblici e alle moderne ricerche della storia della teologia e della storia dei dogmi, il dialogo ecumenico, realizzato dal Concilio Vaticano II in poi, ha contribuito a raggiungere ad una significativa convergenza a riguardo della dottrina della giustificazione. E questi, a sua volta, "permette di formulare in questa Dichiarazione congiunta un consenso su verità fondamentali della dottrina della giustificazione alla luce del quale le condanne dottrinali del XVI secolo ad essa relative oggi non riguardano più la controparte" (DCDG n. 13).

La DCDG non pretende assolutamente che la dottrina luterana della giustificazione sia identica della dottrina cattolica o viceversa. Essa è convinta che "consenso" non significa affermazione dottrinale unica o monolitica, così come unità non è sinonimo di uniformità, come avremo modo di vedere alla fine di questa ricerca. 


\section{La spiegazione della comune comprensione della giustificazione}

Riguardo a una questione dottrinale particolare, come nel nostro caso la dottrina della giustificazione, la ricerca ad un "consenso differenziato", secondo André Birmelé, caporedattore per parte luterana del gruppo di redazione della DCDG, esige un triplice procedimenti:

1. Un accordo su ciò che attiene alla verità fondamentale, che richiede un'affermazione comune sulla questione controversa;

2. una chiarificazione delle esposizioni particolari, che restano diverse e il cui carattere legittimamente differente va precisato e verificato affinché non possa più mettere in discussione l'affermazione fondamentale comune;

3. la conseguenza dei primi due passi, cioè la soppressione degli anatemi pronunciati in passato. Facendo così, quindi, si può giungere all'“unità nella diversità riconciliata” ${ }^{28}$

Ora, vorremmo, proseguire nell'esaminare la DCDG, usando questi tre livelli indicati sopra, per meglio comprendere il "consenso differenziato" raggiunto. La DCDG, infatti, opera in base a questa concezione di un "consenso differenziato" in grado di portare delle differenze teologiche e l'applica alla dottrina della giustificazione. Lo fa in tre tappe che possono essere facilmente distinguibili: L'affermazione fondamentale comune (nn. 14-17); I punti tradizionalmente controversi (nn. 18-39); L'“abolizione” delle condanne e le conseguenze (nn. 40- 44). ${ }^{29}$

\section{L'affermazione fondamentale comune (nn. 14-17)}

Dopo il richiamo del messaggio biblico e l'affermazione di "un consenso su verità fondamentali della dottrina della giustificazione", la DCDG espone, in alcuni brevi paragrafi, la comprensione comune della giustificazione (nn. 14-17). Si può dire che questa parte della DCDG è il cardine e la base di tutto il documento.

A proposito della giustificazione, nel n. 15, troviamo una doppia prospettiva. La giustificazione viene descritta anzitutto a partire da Dio: "Insieme crediamo che la giustificazione è opera di Dio uno e trino." Essa è la volontà di Dio, che per questo manda il suo figlio nel mondo. Mediante lo Spirito Santo le persone partecipano al

\footnotetext{
${ }^{28}$ Birmelé, "Uniti sulla giustificazione", 129.

${ }^{29}$ In questa nostra analisi, seguiamo principalmente alcune osservazioni fatte da A. Birmelé nel suo articolo "Uniti sulla giustificazione" e da W. Kasper nella sua opera Cosechar los frutos. A proposito, cfr. Ibid., 127-136; 57-72.
} 
Cristo che è la loro giustizia. Essendo l'opera del Dio trinitario, la giustificazione avviene "solo per grazia". A partire dalla prospettiva umana, la giustificazione viene descritta mediante la sola nozione della fede, la sola risposta possibile all'iniziativa esclusivamente divina. La DCDG, infatti, osserva che

...tutti gli uomini sono chiamati da Dio alla salvezza in Cristo. Soltanto per mezzo di lui noi siamo giustificati dal momento che riceviamo questa salvezza nella fede. La fede stessa è anch'essa dono di Dio per mezzo dello Spirito Santo che agisce, per il tramite della Parola e dei sacramenti, nella comunità dei credenti, guidandoli verso quel rinnovamento della vita che Dio porta a compimento nella vita eterna. (DCDG n. 16).

La fede è la relazione viva che unisce il credente al Signore. Essa è suscitata dalla Parola e dai sacramenti nella comunità dei credenti, ovvero nella Chiesa. Questa comunità non è un dato ulteriore che verrebbe ad aggiungersi alla giustificazione, ma è parte integrante dell'evento stesso della giustificazione. Nel n. 17, si ricorda che il messaggio della giustificazione non è nient'altro che il messaggio dell'azione salvifica di Dio in Cristo proclamato dal NT. In realtà, la DCDG afferma:

Condividiamo anche la convinzione che il messaggio della giustificazione ci orienta in modo particolare verso il centro stesso della testimonianza che il Nuovo Testamento dà dell'azione salvifica di Dio in Cristo: essa ci dice che noi, in quanto peccatori, dobbiamo la nostra vita nuova soltanto alla misericordia di Dio che perdona e che fa nuove tutte le cose, misericordia che noi possiamo ricevere soltanto come dono nella fede, ma che non possiamo meritare mai e in nessun modo. (DCDG n. 17).

Questa confessione comune potrebbe essere considerata sufficiente e capace di portare sviluppi diversi ma non divergenti. Ma la DCDG non si accontenta di questo. In realtà, essa ha un altro passo da fare, ossia un livello complementare.

\section{I punti tradizionalmente controversi (nn. 18-39)}

Nel secondo livello si riprendono in rapida successione i punti particolari che hanno portato alle condanne fra le due comunità. A dire il vero, questa è la sezione più ampia della DCDG (nn. 18-39); ed essa intende verificare la realtà del consenso fondamentale, riprendendo sette ambiti che sono stati in passato i principali luoghi controversi in relazione alla dottrina della giustificazione: Incapacità e peccato dell'uomo di fronte alla giustificazione; giustificazione come perdono dei peccati e azione che rende giusti; giustificazione mediante la fede e per grazia; l'essere peccatore del giustificato; la Legge e il Vangelo; la certezza della salvezza; le buone opere del giustificato. 
Su ognuno di questi ambiti la DCDG espone tre passi principali: (1) Un consenso fondamentale generale; (2) un nuovo consenso fondamentale su tale ambito; (3) gli sviluppi particolari di ciascuna tradizione su tale ambito. Per spiegare meglio questo, si può dire che su ognuno di questi ambiti la DCDG propone una nuova affermazione comune alle due tradizioni, oltre quella affermazione fondamentale comune. In altri termini, il consenso fondamentale generale viene tradotto, a livello delle affermazioni particolari, in un secondo consenso fondamentale riguardante quel determinato ambito che era stato oggetto di anatema.

Solo dopo aver acquisito questa nuova affermazione comune, la DCDG presenta gli sviluppi particolari di ciascuna tradizione, sformandosi di dimostrare che questi sviluppi, che espongono opzioni teologiche diverse e non dovute solo a categorie mentali e terminologie diverse, esprimono differenze legittime, poiché sono sostenute da un doppio consenso: il consenso fondamentale generale e il consenso fondamentale sulla questione particolare.In realtà,

...questo doppio consenso viene considerato sufficiente al punto che anche le esatte formulazioni già condannate dall'altra tradizione possono essere ora riprese nell'esposizione particolare dei luterani o dei cattolici senza che la controparte abbia nulla da ridire, dal momento che attualmente il doppio consenso precisa il quadro in cui una simile affermazione è possibile e accettabile per la controparte. Così nelle esposizioni luterani della $D C G$ vengono riprese quattro affermazioni condannate dal concilio di Trento e nell'esposizione cattolica viene ripresa una convinzione cattolica rigettata dalla Formula di concordia luterana. ${ }^{30}$

A questo punto, vorremmo dare un esempio concreto, per illustrare questo modo di procedere. L'esempio è quello della questione della "certezza della salvezza":

1. Consenso fondamentale generale. La DCDG si riferisce anzitutto al consenso generale sulla giustificazione (nn. 15-17). In questi passi si afferma, come abbiamo visto, che la giustificazione è opera di Dio uno e trino; e essa avviene "solo per grazia", mediante la fede.

2. Consenso fondamentale su tale questione particolare. Nell'esposizione di questa questione particolare (“certezza della salvezza”), la DCDG, nel n. 34, giunge a un secondo consenso fondamentale:

${ }^{30}$ Birmelé, “Uniti sulla giustificazione”, 131. 
Insieme confessiamo che $\mathrm{i}$ credenti possono fare affidamento sulla misericordia e sulle promesse di Dio. Anche nella loro debolezza e nelle molteplici minacce che mettono in pericolo la loro fede, essi possono contare, in forza della morte e della resurrezione di Cristo, sulla promessa efficace della grazia di Dio nella Parola e nel sacramento ed essere così certi di questa grazia.

Ciò significa che per dare fondamento alla verità della "certezza della salvezza", si mette l'accento sull'affidamento nella misericordia di Dio e nelle sue promesse.

3. Gli sviluppi particolari di ciascuna tradizione. Dopo aver esposto questo, e solo sulla base di questo secondo livello di consenso, vengono esposte gli sviluppi particolari di ciascuna tradizione.

= L'esposizione luterana, nel n. 35:

I riformatori hanno accentuato in modo particolare il fatto che, nella prova, il credente non deve rivolgere lo sguardo a se stesso, ma a Cristo e fare affidamento in modo totale soltanto su di lui. Riponendo così la sua fiducia nella promessa di Dio, egli è certo della sua salvezza, mentre non ne è mai certo se guarda a se stesso.

= L'esposizione cattolica, nel n. 36:

I cattolici possono condividere l'orientamento dei riformatori che consiste nel fondare la fede sulla realtà oggettiva della promessa di Cristo, a prescindere dalla personale esperienza e nel confidare unicamente nella promessa di Cristo (cfr. Mt 16, $19 ; 18,18$ ). Con il Concilio Vaticano II, i cattolici affermano che credere significa abbandonarsi interamente a Dio, che ci libera dalle tenebre del peccato e della morte e ci desta alla vita eterna. In questo senso l'uomo non può credere in Dio e contemporaneamente ritenere che la sua promessa non è affidabile. Nessuno può dubitare della misericordia di Dio e del merito di Cristo, allorché ciascuno può temere per la sua salvezza se considera le sue debolezze e le sue mancanze. Il credente, pur conoscendo i suoi fallimenti, può essere certo che Dio vuole la sua salvezza.

Si può evidentemente notare che le esposizioni teologiche delle due tradizioni rimangono diverse. Ma la DCDG pensa che la convergenza è attualmente sufficiente e che si può parlare di un "consenso differenziato" su questa questione particolare, un consenso che permette ai luterani di parlare di certezza della salvezza senza che la controparte cattolica se ne adombri. Le due accentuazioni, quindi, sono al tempo stesso diverse e aperte l'una all'altra.

Dobbiamo dire finalmente che questa triplice operazione (un consenso fondamentale generale, un nuovo consenso fondamentale su tale ambito e gli sviluppi particolari di ciascuna tradizione su tale ambito) viene applicata anche sugli altri sei ambiti, che sono menzionati sopra, e che sono stati in passato i principali luoghi controversi in riferimento alla dottrina della giustificazione (cfr. $D C D G$, nn. 19-39). 
L'abolizione" delle condanne e le conseguenze (nn. 40-44)

Avendo raggiunto questo doppio consenso (sull'affermazione fondamentale comune e sui punti tradizionalmente controversi), la DCDG passa al terzo livello, l'"abolizione" delle condanne reciproche o degli anatemi. Ciò è la diretta conseguenza dei primi due livelli. La DCDG ritiene che ora sia possibile abolire le condanne reciproche, ovvero affermare che l'insegnamento dell'altra tradizione presente in questa Dichiarazione non è più colpito dalle condanne formulate dalla controparte. In questo senso, la DCDG afferma:

Con ciò, le condanne dottrinali del XVI secolo, nella misura in cui esse si riferiscono all'insegnamento della giustificazione, appaiono sotto una nuova luce : l'insegnamento delle Chiese luterane presentato in questa Dichiarazione non cade sotto le condanne del Concilio di Trento. Le condanne delle Confessioni luterane non colpiscono l'insegnamento della Chiesa cattolica romana così come esso è presentato in questa Dichiarazione. (DCDG n. 41).

È da notare lo stretto legame posto dalla DCDG tra l'abolizione delle condanne reciproche e l'affermazione del consenso. In realtà, i due livelli di affermazioni comuni e l'abolizione degli anatemi sono inseparabili e si condizionano reciprocamente. In effetti, nella loro complementarità e nella loro interdipendenza, essi sostituiscono il consenso. È per questo che la DCDG, nei nn. 40-41, chiedono alle chiese di approvare contemporaneamente entrambe le cose: la Dichiarazione del consenso e l'abolizione degli anatemi. Inoltre, si nota chiaramente che la conclusione della DCDG è breve e si riassume in una triplice affermazione:

1. Un consenso". Esiste "un consenso tra luterani e cattolici su verità fondamentali di tale dottrina della giustificazione" (DCDG n. 40). In realtà, la conclusione ricapitola così i diversi livelli di consenso, di cui abbiamo parlato sopra. Questo consenso, però, non è monolitico, ma include legittime differenze che non possono essere ridotte esclusivamente a questioni di linguaggio, ma che esprimono forme teologiche e accentuazioni particolari.

2. Un "consenso differenziato". Anche se la DCDG non lo dice espressamente, c'è qui un "consenso differenziato". Questi, infatti, include l'affermazione secondo cui l'insegnamento di ciascuna tradizione presente nella DCDG non è più colpito dalle condanne del concilio di Trento e delle confessioni luterane (DCDG n. 41). A dire il vero, le condanne del passato non sono quindi prive di fondamento, ma conservano il significato di "salutari avvertimenti" per ogni insegnamento e ogni pratica ecclesiale (DCDG n. 42).

3. Un "passo decisivo". In realtà, la DCDG non risolve tutto. Essa intende di essere solamente, come lo dichiara al n. 44, "passo decisivo verso il superamento della 
divisione ecclesiale. Preghiamo lo Spirito Santo affinché egli continui a guidarci verso quell'unità visibile che è la volontà di Cristo". Le controversie teologiche, in modo particolare sulla ecclesiologia, sui sacramentali e sui ministeri, non saranno risolte, quindi, da quest' unico consenso sulla giustificazione, ma non potranno neppure essere risolte senza di esso. Il consenso sulla giustificazione raggiunto nella DCDG dovrà essere tradotto, allora, in tutti gli altri ambiti in cui permangono delle differenze che dividono le chiese, come lo dice il documento stesso:

Il nostro consenso su verità fondamentali della dottrina della giustificazione deve avere degli effetti e trovare un riscontro nella vita e nell'insegnamento delle Chiese. Al riguardo permangono ancora questioni, di importanza diversa, che esigono ulteriori chiarificazioni. Esse riguardano, tra l'altro, la relazione esistente tra Parola di Dio e insegnamento della Chiesa, l'ecclesiologia, l'autorità nella Chiesa e la sua unità, il ministero e i sacramenti, ed infine la relazione tra giustificazione e etica sociale. Siamo convinti che la comprensione comune da noi raggiunta offra la base solida per detta chiarificazione. Le Chiese Luterane e la Chiesa Cattolica si adopereranno ad approfondire la comprensione comune esistente affinché essa possa dare i suoi frutti nell'insegnamento e nella vita ecclesiale. (DCDG n. 43).

\section{La Dichiarazione congiunta nella prospettiva ecumenica \\ Un consenso differenziato raggiunto tra la Federazione delle Chiese Luterani e la Chiesa Cattolica}

Ora, dobbiamo trattare la questione del "consenso differenziato" raggiunto tra cattolici e luterani riguardante la dottrina della giustificazione. In questo punto, si vuole valutare da vicino la DCDG, per rispondere a tante questioni: Che novità ha aggiunto al cammino ecumenico questa Dichiarazione? Che senso ha tale Dichiarazione alla molto ricercata "unità visibile della Chiesa"?

Innanzitutto, dobbiamo dire con A. Birmelé che "il documento è stato preparato per decenni attraverso un dialogo teologico ed ecumenico condotto da specialisti. Tuttavia con la Dichiarazione congiunta abbiamo raggiunto una nuova qualità". ${ }^{31}$ Questa nuova qualità consiste principalmente nel fatto che si passa da un "consenso di teologi" a un "consenso di Chiese". Per spiegare meglio questo fatto, si può dire che si passa dai testi conclusivi dei dialoghi, frutto del lavoro dei teologi e pubblicati sotto la loro sola responsabilità, a una dichiarazione ufficiale delle chiese che hanno tutt'altro statuto e tutt'altra autorità. ${ }^{32}$

\footnotetext{
${ }^{31}$ Ibid., 132.

${ }^{32}$ Ibid.,127-128.
} 
A proposito del "consenso", come abbiamo visto, la DCDG osserva al n. 40 cheesiste "un consenso tra luterani e cattolici su verità fondamentali di tale dottrina della giustificazione". Ma "il raggiungimento del consenso non è ancora unità. Perciò, nel processo dell'unione il consenso è una forma iniziale della comunione piena e visibile". ${ }^{33}$ Inoltre, si tratta di un consenso "su verità fondamentali", e non "sulle verità fondamentali", perché potrebbero esistere altre verità fondamentali riguardano alle quali non vi è, o non vi è ancora, consenso. ${ }^{34}$

A dire il vero, la comprensione del consenso e dei suoi diversi livelli proposto dalla DCDG scaturisce da un'esigenza teologica ed ecclesiale. Al termine di tanti anni di lavoro, il dialogo luterano-cattolico non propone un'esposizione teorica sulla concezione del "consenso", ma l'illustra attraverso la sua applicazione concreta sulla dottrina della giustificazione. In realtà,

...gli autori della DCDG erano convinti che la sua applicazione [della concezione del "consenso"] a quest'ambito [alla dottrina della giustificazione] poteva aprire nuove prospettive negli ambiti in cui il dialogo rimane più delicato: l'ambito delle opzioni ecclesiologiche, sacramentali e ministeriali. ${ }^{35}$

Le parole seguenti del cardinale Kasper spiegano in che modo si debba comprendere il "consenso differenziato":

Che esiste un accordo fondamentale sulla dottrina della giustificazione. Evidentemente sussistono sull'argomento alcune questioni aperte che dovranno essere ulteriormente esaminate. Le differenze non annullano tuttavia la base comune che è stata raggiunta sulla comprensione di tale dottrina. Per tanto si tratta anche di un consenso differenziato. ${ }^{36}$

Si può, quindi, concludere che non si tratta di due posizioni (quella cattolica e quella luterana) in sé inconciliabili, ma di due approcci e di due accentuazioni complementari. Per la DCDG le due posizioni non sono esclusive, ma "aperte l'una all'altra", sono anzi dei correttivi necessari che permettono di evitare le tentazioni specifiche di ognuna di queste posizioni. La dottrina della giustificazione, infatti, non è più il grande problema teologico che divide tra i cattolici e luterani. Ma è comunque innegabile che essi non condividono una sola e stessa dottrina della giustificazione.

\footnotetext{
${ }^{33}$ Lehmann, "Fondamentalmente uniti", 709.

${ }^{34}$ Ibid., 709.

${ }^{35}$ Birmelé, "Uniti sulla giustificazione”, 132.

${ }^{36}$ Kasper, "La Dichiarazione congiunta sulla dottrina della giustificazione", 10.
} 
È molto importante dire che la traduzione ecclesiologica di quest'accordo sulla comprensione della giustificazione è invece più delicata. La DCDG, al n. 43, ricorda certe controversie tradizionali (ecclesiologiche, sacramentali e ministeriali) sono ancora di natura tale da dividere le Chiese. Anche se in tutti questi ambiti menzionati sono stati fatti notevoli passi, non si può ancora parlare di un sufficiente consenso differenziato, tale da permettere i passi decisivi per l'unità visibile della Chiesa. Ma la DCDG pensa giustamente che l'accordo raggiunto sulla giustificazione può ora permettere di affrontare le questioni teologiche e ecclesiologiche controversie in modo nuovo (cfr. DCDG n. 43).

\section{Alcune reazioni sulla Dichiarazione congiunta}

Dopo aver cercato di capire il significato del "consenso differenziato" sulla dottrina della giustificazione, vorremmo ora raccogliere brevemente alcune reazioni sulla DCDG. In realtà, la critica teologica ha riguardato non tanto il contenuto e la comprensione della dottrina della giustificazione quanto piuttosto la definizione del "consenso differenziato" proposto e applicato dalla DCDG. Sono principalmente due tipi di risposta che sono concentrate sul "consenso differenziato" tra cattolici e luterani: la risposta di un "neo-fondamentalismo confessionalista" e la risposta basata su un argomento teologico. ${ }^{37}$

\section{La risposta di un "neo-fondamentalismo confessionalista"}

La prima risposta che possiamo chiamare come un "neo-fondamentalismo confessionalista" è stata avanzata da alcune persone sia tra i cattolici sia tra i luterani. Essi hanno constatato una mancanza d'identità fra le affermazioni dottrinali luterane e cattoliche della DCDG e quelle delle confessioni di fede o dei riferimenti conciliari, perciò hanno criticato negativamente il consenso raggiunto. Questa risposta non propone l'abolizione delle condanne storiche reciproche. Essi hanno notato l'esistenza di convergenze importanti su verità fondamentali, ma non trae le conseguenze proposte dalla DCDG.

Si può dire che la prima risposta del Vaticano intitolata Risposta sulla giustificazione (1998), da parte del Pontificio Consiglio per l'Unità dei Cristiani, è una risposta di questa natura. In fatti, nella risposta, si compara direttamente le conclusioni della DCDG con quelle del Concilio di Trento. Dopo aver sottolineato gli

${ }^{37}$ Per presentare queste due tipi di risposte, si basa principalmente su Birmelé, "Uniti sulla giustificazione", 127-136. 
avvicinamenti significativi, il testo aggiunge: "La Chiesa Cattolica ritiene tuttavia che non si possa ancora parlare di consenso tale che elimini ogni differenza fra i cattolici e i luterani nella comprensione della giustificazione." ${ }^{38} \mathrm{Si}$ affermava che i luterani non sono ancora diventati tridentini.

Tra i luterani, poi, ci sono sorti certi professori protestanti tedeschi che hanno risposto negativamente alla DCDG. In un manifesto, firmato da circa 150 teologi, essi hanno dichiarato che non vi è consenso sulla salvezza per sola fede, sulla certezza della salvezza, sull'essere peccatore del giustificato, sul significato delle opere buone, e su altre questioni. Si nota anche qui che i teologi autori del manifesto sembrano aver proiettato nella DCDG una comprensione del consenso-uniformità che la DCDG desiderava precisamente oltrepassare. Essi hanno semplicemente comparato la DCDG con gli scritti confessionali luterani e constatato che i cattolici non sono ancora diventati luterani.

$\grave{E}$ evidente che in queste risposte e reazioni, i riferimenti alla teologia contemporanea in tutto il dibattito avvenuto in Germania siano assenti totalmente. E per dirla con le parole di A. Birmelé:

Nessun commento -e sono stati numerosi- prende in considerazione un'evoluzione del luteranesimo negli ultimi cinque secoli. La $D C G$ viene letta come se partecipasse direttamente ai colloqui di Ratisbona del 1541. Con questa griglia di lettura le conclusioni sono scontate, ed è molto facile dimostrare che le concezioni della Riforma luterana e del concilio di Trento divergevano e si contrapponevano in modo pressoché radicale. La DCG argomenta deliberatamente a partire dalla situazione contemporanea. Essa non riprende gli argomenti del XVI secolo così come venivano avanzati allora, non perché li considera falsi, ma perché è intervenuta un'evoluzione delle identità ecclesiali. ${ }^{39}$

Si può affermare che il "consenso differenziato", come si è detto in diversi modi, non esige l'uniformità dottrinale, ma è aperto a opzioni teologiche differenti.

\section{La risposta basata su un argomento teologico}

La seconda risposta, che possiamo chiamare la "risposta basata su un argomento teologico", da una parte parla della "mancanza delle conseguenze ecclesiologiche"; d'altronde, parla del "problema criteriologico". Questa risposta, che tratteremo brevemente, interessa in modo particolare la nostra ricerca che tenterà alla fine di tirare una visione ecclesiale in prospettiva ecumenica che emerge dalla DCDG.

\footnotetext{
${ }^{38}$ Pontificio Consiglio per l'Unità dei Cristiani, "Risposta sulla giustificazione”, 497.

${ }^{39}$ Birmelé, "Uniti sulla giustificazione”, 133.
} 
La mancanza delle conseguenze ecclesiologiche. Questo primo tipo di risposta basato su un argomento teologico, ovvero la "mancanza delle conseguenze ecclesiologiche", è proposto da $\mathrm{E}$. Jünge $\mathrm{l}^{40}$. E questo argomento sarà ripreso dalle chiese luterane tedesche che eviteranno di chiamare consenso l'avvicinamento proposto dalla DCDG. In sostanza, Jüngel contesta DCDG e lamenta il fatto che questo consenso proposto dalla DCDG non abbia delle conseguenze ecclesiologiche.

Poiché nel luteranismo la giustificazione non è un dato dottrinale fra gli altri, ma il criterio di tutta la vita ecclesiale, è per lui (per Jüngel) inconcepibile affermare un consenso sulla giustificazione senza trarne immediatamente delle conseguenze ecclesiologiche. Ai tre livelli complementari, che costituiscono il "consenso differenziato" proposto dalla $D C G$, Jüngel vorrebbe aggiungere un quarto: le conseguenze ecclesiologiche. ${ }^{41}$

Si può comprendere tale difficoltà dal momento che la DCDG è molto prudente riguardo a queste conseguenze ecclesiologiche. La DCDG, infatti, propone di chiarirle ulteriormente:

Al riguardo permangono ancora questioni, di importanza diversa, che esigono ulteriori chiarificazioni. Esse riguardano, tra l'altro, la relazione esistente tra Parola di Dio e insegnamento della Chiesa, l'ecclesiologia, l'autorità nella Chiesa e la sua unità, il ministero e i sacramenti, ed infine la relazione tra giustificazione e etica sociale (DCDG n. 43).

Dall'altra parte, però, si deve notare due cose fondamentali. La prima riguarda il termine "Chiesa". Parlando di "le Chiese luterane e la Chiesa cattolica", al n. 1835, si fa una nota (la nona nota) in cui la DCDG afferma che usando il termine "Chiesa" non pretende di risolvere le questioni ecclesiologiche:

Nella presente Dichiarazione il termine "Chiesa" è adoperato nel senso secondo il quale esso è compreso da ciascuna delle due Chiese coinvolte nel dialogo, senza alcuna pretesa di risolvere le questioni ecclesiologiche che a detto termine sono collegate. ${ }^{42}$

La seconda cosa riguarda il senso dell'abolizione degli anatemi. Si potrebbe certamente aggiungere che un' eventuale abolizione delle condanne e delle anatemi da parte

\footnotetext{
${ }^{40}$ Eberhard Jüngel è nato a Magdeburgo (Germania) nel 1934. È un discepolo di K. Barth e di G. Ebeling. La sua opera più importante è intitolata Dio, mistero del mondo. Per una fondazione della teologia del Crocifisso nella disputa fra teismo e ateismo. Cfr. Semeraro, "Jüngel Eberhard", 720-721.

${ }^{41}$ Birmelé, "Uniti sulla giustificazione", 133.

${ }^{42}$ Cereti e Puglisi (cur.), Enchiridion oecumenicum 7, 1835; 887.
} 
del Vaticano avrebbe un significato ecclesiologico importante, perché esprimerebbe un certo riconoscimento del carattere ecclesiale del luteranesimo. Nonostante tutte queste annotazioni, l’argomento di Jüngel rimane valido. Al riguardo, A. Birmelé osserva:

Gli estensori della $D C G$ avrebbero evidentemente auspicato che l'affermazione del consenso avesse delle conseguenze ecclesiologiche immediate, alla stregua delle diverse dichiarazioni di comunione fra le Chiese derivate dalla Riforma. Ma vi hanno rinunciato, poiché sapevano che il dialogo luterano-cattolico non era ancora in grado di farlo. E tuttavia erano convinti che fosse possibile già ora una tappa intermedia. Essa sembrava loro più realistica. ${ }^{43}$

Si può notare, poi, che Jüngel ed insieme a lui le chiese luterane tedesche non rifiutano la comprensione del consenso proposto dalla DCDG, ma la considerano incompleta, perché mancano le conseguenze ecclesiologiche che modificano fondamentalmente le relazioni tra i luterani e i cattolici. In fine, a causa del legame tra questo argomento teologico e la nostra ricerca ad un visione ecclesiale in prospettiva ecumenica dentro la DCDG, riprenderemo alla fine la discussione su tale questione.

Il "problema criteriologico". Inoltre, lo stesso Jüngel e altri professori protestanti tedeschi accanano ad un altro problema, ovvero il cosiddetto il "problema criteriologico". Esso può essere riassunto essenzialmente in questa domanda: la dottrina della giustificazione è "un criterio" o "il criterio" di tutta la dottrina e la prassi della Chiesa?

Si può dire che il nodo teologico del problema è quello del posto della soteriologia nell'esposizione dell'insegnamento cristiano, un posto che tutti affermano essere particolare, senza tuttavia accordarsi sul suo carattere unico e sulla sua unicità. A proposito della funzione criteriologica della dottrina della giustificazione, e come risposta al problema criteriologico, la DCDG aveva osservato:

Pertanto, la dottrina della giustificazione che assume e sviluppa tale messaggio, non è soltanto una singola parte dell' insegnamento di fede cristiano. Essa si pone in una relazione essenziale con tutte le verità della fede che vanno considerate interiormente connesse tra loro. Essa è un criterio irrinunciabile che orienta continuamente a Cristo tutta la dottrina e la prassi della Chiesa. Quando i luterani sottolineano il significato del tutto singolare di questo criterio, essi non negano la connessione e il significato di tutte le verità di fede. Quando i cattolici si sentono vincolati da molteplici criteri, non per questo negano la particolare funzione del messaggio della giustificazione. Luterani e cattolici tendono insieme alla meta di confessare in ogni cosa Cristo, il solo nel quale riporre ogni fiducia, poiché egli è l'unico mediatore (1 Tm 2, 5s) attraverso il quale Dio nello Spirito Santo fa dono di sé e effonde i suoi doni che tutto rinnovano. (DCDG n. 18).

${ }^{43}$ Ibid., 133-134. 
Tuttavia si può affermare giustamente con A. Birmelé che

...questo paragrafo susciterà un lungo e difficile dibattito, che è ben lungi dall'essere concluso, non solo nel dialogo fra le due tradizioni firmatarie della DCDG, ma anche all'interno di ciascuna di esse e più particolarmente in seno alle Chiese originate dalla Riforma. ${ }^{44}$

In realtà, a proposito dell'affermazione della DCDG, la parte cattolica continua a difendere la scelta fatta dalla DCDG. Kasper, infatti, ha tentato di giustificare tale scelta, insistendo sull'espressione di "un criterio indispensabile". Nessuna opzione ecclesiale o dottrinale della Chiesa Cattolica può fare a meno di questo criterio che esige una "focalizzazione cristologica". Per ciò egli difende l'espressione "un criterio", perché "i cattolici si sentono vincolati da molteplici criteri". ${ }^{45}$ Inoltre, non si può affermare l'esclusività della dottrina della giustificazione per esporre il messaggio della salvezza. ${ }^{46}$

Ma la reazione di alcuni professori luterani, in modo particolare Jüngel, invece, va verso la volontà di affermare l'esclusività della dottrina della giustificazione per l'ermeneutica ed il giudizio di tutte le verità di fede. Perciò, tutte le affermazioni dottrinali della Chiesa devono essere sottoposte a questo criterio.Certamente, la questione è molto seria, e non si può ridurla ad alcuni affermazioni o critiche; ma qui "occorre - dice Birmelé- ripetere che la DCDG era ben consapevole di questa difficoltà e il n. 18 non afferma assolutamente che il posto del messaggio della giustificazione nell'insieme dell'insegnamento cristiano sia lo stessa nelle due tradizioni. ${ }^{47}$

Dopo questa sintesi del panorama delle reazioni suscitate sulla DCDG, si notano chiaramente le sfide metodologiche che hanno causato le diverse reazioni: la concezione del "consenso differenziato" e il "problema del criterio". In realtà, il Concilio Vaticano II, da parte sua, riconosce un "ordine o gerarchia nelle verità della dottrina cattolica, essendo diverso il loro nesso con il fondamento della fede cristiana" ${ }^{48} \mathrm{Si}$ può anche dire che la DCDG propone di affermare il consenso sulla giustificazione, anche se la funzione criteriologica e le conseguenze ecclesiale restano

\footnotetext{
${ }^{44}$ Ibid., 134.

${ }^{45} \mathrm{Si}$ può pensare, per esempio, alla tradizione e alla storia della Chiesa, al ministero e alla comprensione della Chiesa.

${ }^{46}$ Kasper, "La Dichiarazione congiunta sulla dottrina della giustificazione", 7-14.

${ }^{47}$ Birmelé, "Uniti sulla giustificazione", 134.

${ }^{48}$ Concilio Vaticano II, “Decreto sull'ecumenismo Unitatis redintegratio" 11, Vatican, http://www. vatican.va/archive/hist_councils/ii_vatican_council/documents/vat-ii_decree_19641121_unitatisredintegratio_it.html (consultato 20 di aprile 2015).
} 
aperte (cfr. DCDG, n. 43). Questa scelta, come dice la stessa DCDG al n. 44, è quella di "passo decisivo verso il superamento della divisione ecclesiale", ossia di una tappa intermedia nel cammino ecumenico.

\section{Uno sguardo allo stato attuale dell'ecumenismo dopo la Dichiarazione congiunta}

A questo punto, partendo dal "consenso differenziato" proposto dalla DCDG, desideriamo descrivere brevemente e generalmente lo stato attuale del cammino ecumenico; cercheremo cioè di raccogliere i fondamentali punti che riguardano il dialogo ecumenico attuale, in modo particolare tra cattolici e luterani, dopo la DCDG.

\section{Il dialogo ecumenico nel ventesimo secolo}

Il movimento ecumenico ${ }^{49}$, come è ben noto, è iniziato prima dell'apertura del Concilio Vaticano II; ma esso trova in tale Concilio la sua spinta più grande. Infatti, dice lo stesso concilio nel Decreto sull'ecumenismo Unitatis redintegratio: "Promuovere il ristabilimento dell'unità fra tutti i cristiani è uno dei principali intenti del sacro Concilio ecumenico Vaticano II." ${ }^{\text {50 }}$

Seguendo con fedeltà e slancio le indicazioni del concilio, la Chiesa Cattolica ha progredito senza sosta nel cammino dell'ecumenismo. Essa, con altre chiese e comunità ecclesiali, ha cercato l'ecumenismo in distinte vie: la via del dialogo teologico, la via dell'ecumenismo spirituale e la via del servizio comune. ${ }^{51}$

Secondo il cardinale Kasper, il ventesimo secolo sarà chiamato "il secolo del risveglio ecumenico": "Oltre ad essere ricordato per i molti eventi terribili, questo ventesimo secolo passerà alla storia come il secolo del risveglio ecumenico." ${ }^{52}$ In questo ventesimo secolo si situa anche la DCDG e le sue conseguenze al cammino ecumenico.

\footnotetext{
${ }^{49}$ A proposito della questione dell'ecumenismo, del suo iter storico, della sua esperienza e della sua dottrina, si veda l'articolo sintetico di Pattaro, "Ecumenismo", 349-370.

${ }^{50}$ Concilio Vaticano II, “Decreto sull'ecumenismo Unitatis redintegratio" 1, Vatican, http://www.vatican. va/archive/hist_councils/ii_vatican_council/documents/vat-ii_decree_19641121_unitatis-redintegratio_ it.html (consultato 20 di aprile 2015).

51 "Molteplici sono anche le manifestazioni dell'incontro ecumenico a livello di ricerca teologica, di preghiera comune e celebrazione del culto, di cooperazione e progetti finalizzati a lenire i bisogni esistenti in varie parti del mondo, di risposte comuni alle sfide del nostro tempo." (Track, "Limportanza della Dichiarazione congiunta", 713).
}

${ }^{52}$ Kasper, "La Dichiarazione congiunta sulla dottrina della giustificazione", 7. 
Si può quindi affermare che tale documento è un grande segno di questo risveglio ecumenico che in cammino fino ai nostri giorni. In realtà, tale Dichiarazione ha una grande rilevanza a causa almeno di due motivi: Essa è firmata ufficialmente dalle chiese e poi essa consente di superare gli anatemi reciproci fulminati dalle chiese proprio sulla questione della giustificazione. Perciò, la DCDG "ha ridato nuova vita e speranza a tutto il movimento ecumenico al termine del secondo millennio dell'era cristiana". ${ }^{53}$

\section{Il dialogo ecumenico attuale tra cattolici e luterani}

Innanzitutto, si afferma che "l'ecumenismo non è pertanto una scelta opzionale, ma è un sacro obbligo" ${ }^{54}$ E questo ecumenismo si basa certamente sulla verità e sulla carità che sono intimamente connesse e non possono sostituirsi a vicenda. Ma l'altra cosa da osservare è l'opera dello Spirito santo in questo cammino ecumenico: "È con grande gratitudine che dobbiamo riconoscere in tale sviluppo (dell'ecumenismo) l'agire dello Spirito che guida la Chiesa." ${ }^{55}$

A proposito dei gruppi che partecipano al dialogo ecumenico attuale, risulta illuminante la distinzione che fa il cardinale Kasper davanti al Colleggio Cardinalizio (2007). Esso individua tre campi nell'ecumenismo: (1) Le relazioni con le antiche Chiese orientali e con le Chiese ortodosse del primo millennio ${ }^{56}$; (2) le relazioni con le comunità ecclesiali nate direttamente o indirettamente -come le Chiese libere-della Riforma del XVI; (3) le relazioni col movimento carismatico e col movimento pentecostale ${ }^{57}$, sorti all'inizio del XX secolo e diffusisi nel frattempo in tutto il mondo con una crescita esponenziale. ${ }^{58}$

Parlando, poi, delle relazioni con le comunità ecclesiali nate dalla Riforma del XVI, Kasper dice che "un certo consenso è stato raggiunto nell'ambito delle verità di fede, soprattutto per ciò che riguarda le questioni fondamentali della dottrina sulla giustificazione. In molti luoghi esiste una fruttuosa collaborazione nella sfera sociale e

\footnotetext{
${ }^{53}$ Cassidy, "Continuare la costruzione", 712.

${ }^{54}$ Kasper, "Tutti siano una cosa sola”, 1.

55 Ibid., 2.

${ }^{56}$ I temi discussi con le Chiese Orientali e Ortodosse sono concentrati su diversi campi teologici: cristologia, ecclesiologia, sacramenti, mariologia e il primato del vescovo di Roma. Cfr. Ibid., 3.

57 "Con circa 400 milioni carismatici e pentecostali, sono al secondo posto tra le comunità cristiane in termini numerici e conoscono una crescita esponenziale." (Ibid., 4).

${ }^{58}$ Ibid.
} 
umanitaria". ${ }^{59}$ Però, a giudizio ancora di Kasper, anche se c'è una fiducia reciproca ed amicizia tra i cattolici e luterani, questo non esclude delle difficoltà e delle delusioni verificate: "Si è diffuso progressivamente un atteggiamento di fiducia reciproca e di amicizia, caratterizzante da un profondo desiderio di unità, che rimane tale nonostante ci siano, di tanto in tanto, toni più duri e aspre delusioni." ${ }^{\prime 60}$

Grazie la DCDG, i cattolici e luterani sono pervenuti a un consenso fondamentale sulla dottrina della giustificazione; ma si trovano ora a dover nuovamente discutere di temi controversi classici o moderni. Tra questi temi controversi, Kasper elenca fondamentalmente quarto temi che presenteremo ora brevemente. ${ }^{61} \mathrm{Il}$ primo tra questi temi è soprattutto quello che riguarda l'“ecclesiologia e i ministeri ecclesiali”.

Ma il secondo tema, che è una conseguenza del primo e non meno importante, è lo "scopo dell'ecumenismo". Kasper, infatti, osserva che "le differenti ecclesiologie portano necessariamente ad avere visioni differenti di ciò che è lo scopo dell'ecumenismo. Così è un problema il fatto che ci manchi un concetto comune di unità ecclesiale quale obiettivo da raggiungere". ${ }^{62}$

Il terzo tema riguarda il "campo etico":

Mentre da una parte ci sforziamo di superare le vecchie controversi, dall'altra emergono nuove divergenze nel campo etico. Ciò riguarda in particolare le questioni attinenti alla difesa della vita, al matrimonio, alla famiglia e alla sessualità umana. ${ }^{63}$

Il quarto e l'ultimo tema è il "patrimonio comune riguardante alla Cristologia e alla Trinità":

La teologia protestante, segnata durante i primi anni del dialogo [ecumenico] dalla "rinascita luterana" e dalla teologia della parola di Dio di Karl Barth, è ora ritornata ai motivi della teologia liberale. Di conseguenza, constatiamo che, da parte protestante, quei fondamenti cristologici e trinitari, che erano stati finora un presupposto comune, vengono a volte diluiti. Ciò che ritenevamo essere il nostro patrimonio comune cominciato a sciogliersi qua e là come $\mathrm{i}$ ghiacciai nelle Alpi. ${ }^{64}$

\footnotetext{
${ }^{59}$ Ibid., 3.

${ }^{60}$ Ibid.

${ }^{61} \mathrm{Su}$ questi temi controversi ed altri, si veda anche Idem, Cosechar los frutos31-53; 73-231.

${ }^{62}$ Idem, "Tutti siano una cosa sola", 3.

${ }^{63}$ Ibid., 3-4.

${ }^{64}$ Ibid., 4.
} 
Da tutto ciò, si può notare che la trasformazione sperimentata dalla Chiesa e dal mondo in generale ha una grande influenza nella trasformazione della situazione ecumenica attuale. In realtà, nella società cosiddetta postmoderna, si crescono continuamente il relativismo religioso e la nascita di numerose chiese; e ciò rende indubbiamente il paesaggio ecumenico ora molto differenziato e confuso. In che modo allora proseguire nel cammino ecumenico?

Proponendo alcuni suggerimenti, Kasper ha cercato di offrire un contributo a questa domanda. Esso suggerisce giustamente di "partire dal comune patrimonio di fede e restare fedeli a ciò che con l'aiuto di Dio abbiamo già raggiunto ecumenicamente". Ciò significa partire dai fondamenti di nostra fede: "Una fede salda e consapevole nel Dio viventi, trino e unico, nella divinità di Cristo, nella forza salvifica della croce e della risurrezione." Basando sulla fede comune, si può discutere sulle nostre differenze in un dialogo teologico fruttuoso. In tutto ciò, l'ecumenismo spirituale trova un posto centrale, ovvero "l'ecumenismo della preghiera della conversione del cuore e della santificazione personale". ${ }^{65}$

\section{Conclusione}

Alla fine della nostra ricerca, tenteremo ora di raccogliere gli elementi essenziali che emergono da essa per individuare una visione ecclesiologica ecumenica in quanto questi elementi raccolti ci permettono.

\section{II modello dell'unità ecclesiale}

È evidente che dopo la DCDG rimane tanto lavoro da fare, in modo particolare a proposito delle questioni ecclesiologiche. Al riguardo, rimangono certamente alcune sfide principali, come, per esempio, la visione dell'unità visibile della Chiesa o il modello dell'unità. ${ }^{66}$ Parlando di questo la DCDG è molto esplicita:

Al riguardo permangono ancora questioni, di importanza diversa, che esigono ulteriori chiarificazioni. Esse riguardano, tra l'altro, la relazione esistente tra Parola di Dio e insegnamento della Chiesa, l'ecclesiologia, l'autorità nella Chiesa e la sua unità, il ministero e i sacramenti, ed infine la relazione tra giustificazione e etica sociale. (DCDG n. 43).

\footnotetext{
${ }^{65}$ Ibid., 5; Idem, Cosechar los frutos, 21-53; 233-245.

66 "La domanda è in particolare di ciò che noi intendiamo per unità visibile della Chiesa, unità che costituisce lo scopo del nostro dialogo. Sarebbe importante chiarire quali sono gli elementi necessari all'unità della Chiesa, e dove conviene collocare, nell'ambito di questa unità, la diversità e la libertà." (Kasper, "La Dichiarazione congiunta sulla dottrina della giustificazione", 12).
} 
Sulla mancanza di visione ecclesiale della unità, come abbiamo visto, è soffermato anche il cardinale Kasper. Esso osserva: "Così è un problema il fatto che ci manchi un concetto comune di unità ecclesiale quale obiettivo da raggiungere. ${ }^{\prime 77} \mathrm{Ma}$ nonostante l'assenza di un modello ecumenico di unità, la DCDG, al n. 44, parla di "passo decisivo verso il superamento della divisione ecclesiale". Infatti, "la Dichiarazione in sé una pietra che delimita il confine $e$ una pietra miliare, se si colloca il testo nella storia, verso il passato e verso il futuro". ${ }^{68} \mathrm{La} D C D G$ è "una tappa aperta sul futuro. Possono e devono seguire altri passi". ${ }^{69}$

In realtà, il 31 ottobre 1999 (il giorno della firma della DCDG) è una grande opportunità per il camino verso l'unità ecclesiale. Questa data, come afferma K. Lehmann, "è una tappa intermedia indispensabile e importantissima, che non ci deve paralizzare, ma infondere coraggio e fiducia sulla strada verso l'unità della Chiesa". ${ }^{70}$ E perciò l'accordo raggiunto con la DCDG "cambia già fin d'ora la relazione fra la Chiesa Cattolica romana e le Chiese Luterane. É un passo decisivo verso il reciproco riconoscimento come chiese, verso la presa di coscienza della responsabilità delle chiese cristiane nei riguardi della testimonianza e del servizio al mondo". ${ }^{71}$ Si può concludere, quindi, che la DCDG è "un documento che costituisce un significativo passo avanti verso il ristabilimento dell'unità fra i seguaci di Cristo". ${ }^{72}$

\section{Unità della Chiesa nella diversità riconciliata}

La novità essenziale, a nostro avviso, che la DCDG può contribuire alla ricerca del modello di unità ecclesiale è quella che riguarda la concezione dell'“unità nella diversità riconciliata". Infatti, la DCDG, oltre di essere un "passo decisivo" verso l'unità della Chiesa, offre un modello valido dell'unità tanto desiderata della Chiesa.

La DCDG sta a dire praticamente che "ciò che ci unisce è più forte di ciò che ci distingue o ci divide". ${ }^{73} \mathrm{E}$ le legittime differenze messe nella comunanza di vedute

\footnotetext{
${ }^{67}$ Idem, "Tutti siano una cosa sola", 3.

${ }^{68}$ Lehmann, "Fondamentalmente uniti", 711.

${ }^{69}$ Krause, "Un passo importante", 713.

${ }^{70}$ Lehmann, "Fondamentalmente uniti”, 712.

${ }^{71}$ Track, "L'importanza della Dichiarazione congiunta”, 713.

${ }^{72}$ Cassidy, "Continuare la costruzione", 712.

${ }^{73}$ Krause, "Un passo importante", 713.
} 
sono "l'espressione del fatto che la chiesa è un'unità nella diversità" ${ }^{74}$ In realtà, si può sostenere, senza relativizzare il risultato raggiunto sulla dottrina della giustificazione, che il messaggio essenziale della DCDG è il seguente:

Nonostante la persistenza di differenze nella concezione della chiesa e la mancanza del reciproco riconoscimento come chiesa, già in questo si è realizzato il reciproco riconoscimento della dottrina della chiesa e il riconoscimento come chiese. $^{75}$

A dire il vero, parlando dell'unità della Chiesa, si tratta essenzialmente di una diversità riconciliata e non della conversione dell'altro alla mia confessione. Però, la domanda fondamentale che emerge qui è la seguente: Come è possibile questa unità nella diversità riconciliata? Cercando di indicare quest'unità della Chiesa nella diversità riconciliata, J. Track, afferma:

Partendo dal fondamento comune, cioè dalla confessione del Dio uno e trino e della sua azione salvifica in Gesù Cristo, bisogna provare se può essere possibile riconoscere nella dottrina e nella prassi dell'altra chiesa una comprensione del Vangelo e una forma dell'essere cristiani e dell'essere chiesa che non contraddica fondamentalmente, nonostante tutta la diversità, quella propria. ${ }^{76}$

Dopo aver spiegato e approfondito la sua prospettiva, Track sostiene che se si condivide questa concezione di fondo è possibile arrivare al reciproco riconoscimento della Chiesa cattolica e delle Chiese luterane nella loro diversità piena di tensioni e arricchente. ${ }^{77}$ In realtà, la prospettiva del cardinale Kasper, come abbiamo visto, andava anche verso questa realtà dell'unità nella diversità riconciliata ${ }^{78}$. Inoltre, la Dichiarazione ufficiale comune afferma:

Il dialogo, sulla base del consenso raggiunto, deve continuare particolarmente su quegli argomenti che sono specificati nella Dichiarazione congiunta stessa (DG 43) come questioni che esigono ulteriore chiarimento, in vista di raggiungere la piena comunione ecclesiale, una unità nella diversità nella quale le differenze che permangono sarebbero "riconciliate" e non avrebbero più la forza di dividere. ${ }^{79}$

\footnotetext{
${ }^{74}$ Lehmann, "Fondamentalmente uniti", 710.

${ }^{75}$ Track, "L'importanza della Dichiarazione congiunta", 714.

${ }^{76}$ Ibid., 714.

77 Ibid., 715.

${ }^{78}$ Kasper, "La Dichiarazione congiunta sulla dottrina della giustificazione", 7-14; Idem, "Tutti siano una cosa sola", 1-5.

${ }^{79}$ Dichiarazione ufficiale comune 3.
} 
È chiaro che questa visione dell'unità nella diversità riconciliata sia molto interessante; però, essa deve affrontarsi, per non dire scontrarsi, con diverse dottrine proprie a ciascuna chiesa. Perciò, queste dottrine proprie ed affermazioni della fede di ciascuna chiesa debbano occupare il loro giusto posto e non di più, altrimenti possono diventare un'ideologia o idoli. ${ }^{80}$

\section{La testimonianza comune}

Senza dubbio alcuno, la divisione dei cristiani è un grande scandalo davanti gli occhi degli uomini del nostro tempo. Al riguardo, il Concilio Vaticano II osserva apertamente: "Tale divisione non solo si oppone apertamente alla volontà di Cristo, ma è anche di scandalo al mondo e danneggia la più santa delle cause: la predicazione del Vangelo ad ogni creatura." ${ }^{81}$

Perciò la ricerca all'unità visibile della Chiesa è importante anche perché il mondo possa credere. In questo contesto, si situa certamente il "consenso" raggiunto nella DCDG. Esso è stato un passo importante verso la comune testimonianza dei cristiani nel mondo. In realtà, si può affermare con C. Krause, il presedente della FLM: "Il consenso raggiunto nella parola diventa credibile nell'azione comune e nello sforzo comune delle nostre chiese, là dove noi incontriamo e soccorriamo le persone oppresse da necessità fisiche e spirituali." 82

Qui valgono anche le parole di Giovanni Paolo II dette in occasione del 450 anniversario della Confessio augustana: "La volontà di Cristo e i segni dei tempi ci spingono a una comune testimonianza nella crescente pienezza della verità e dell'amore." ${ }^{83}$ Si può quindi accogliere l'importanza del "consenso" raggiunto nella DCDG nell'ottica della comune testimonianza delle chiese cristiane davanti agli uomini di oggi. Da una parte tale consenso facilita la comune testimonianza nelle circostanze concrete della vita quotidiana; e dalla altra parte, la comune testimonianza spinge i cristiani alla ricerca alla visibile unità della Chiesa.

\footnotetext{
${ }^{80} \mathrm{Nel}$ contesto della sua omelia ai vespri ecumenici (30 ottobre 1999), intitolata "vi raccoglierò, vi radunerò", Kasper osserva: "Anche le affermazioni dottrinali della chiesa, che sono vere e importanti, possono diventare idoli, se non vengono più intese come indicazioni segnaletiche e come promemoria del mistero di Dio, ma diventano fine a se stesse, idolo, feticcio, grido di battaglia. Possiamo sperimentare cose del genere anche ai nostri giorni." (Kasper, "Vi raccoglierò, vi radunerò", 710).

${ }^{81}$ Concilio Vaticano II, "Decreto sull'ecumenismo Unitatis redintegratio" 1, Vatican, http://www.vatican. va/archive/hist_councils/ii_vatican_council/documents/vat-ii_decree_19641121_unitatis-redintegratio_ it.html (consultato 20 di aprile 2015).

${ }^{82}$ Krause, "Un passo importante", 713.

${ }^{83}$ Questo testo è stato citato da C. Krause nella sua omelia. Cfr. Ibid., 713.
} 
La comune testimonianza dei cristiani basata sul "consenso" raggiunto nella DCDG non riguarda solo le necessità materiali e fisiche degli uomini di oggi; ma essa si allarga anche alle necessità spirituali ed il bisogno del perdono e della riconciliazione dell'uomo di oggi e della l'odierna società da parte di Dio. Al riguardo, parlando del processo dialogico che ha condotto alla DCDG, Track osserva:

Consolante in questo processo è il fatto che la dottrina della giustificazione è ritornata a essere un tema affrontato nell'opinione pubblica ecclesiale e sociale. Occorre ritornare a parlare in modo salvifico della concezione della giustificazione e presentarla come una visione che libera, dona la fede, promette il perdono, apre all'amore e fonda la speranza per la nostra vita e per il nostro mondo nel presente e per il presente. Ė questo è il compito che dobbiamo assumere oggi e sempre oggi mediante una testimonianza comune, sapendo che la riuscita può essere solo donata. ${ }^{84}$

In realtà, la Dichiarazione ufficiale comune parla della testimonianza comune in termini simili:

Luterani e cattolici continueranno ad adoperarsi in spirito ecumenico nella loro testimonianza comune per interpretare il messaggio della giustificazione in un linguaggio che sia adatto agli uomini di oggi, e con riferimento a quelle preoccupazioni dei singoli e della società del nostro tempo. ${ }^{85}$

A mo' di conclusione, partendo dalla DCDG, si può affermare che i cattolici e luterani hanno messo d'accordo sui principali punti che facevano delle difficoltà nel passato riguardante la questione della giustificazione. La DCDG è così un passo di grande valore nel cammino ecumenico, perché "essa esprime un consenso su verità fondamentali della dottrina della giustificazione” (DCDG n. 6). Tale consenso può spingere col tempo i cristiani a cercare una visione ecumenica sulla unità della Chiesa, per arrivare un giorno all'unità visibile e piena promessa e tanto voluta da Cristo stesso (Gio 17,11). ${ }^{86}$

\footnotetext{
${ }^{84}$ Track, "L'importanza della Dichiarazione congiunta", 714.

${ }^{85}$ Dichiarazione ufficiale comune, n. 3.

${ }^{86} \mathrm{Nel}$ contesto della sua breve omelia nel corso della solenne celebrazione della ratificazione della DCDG (31 ottobre 1999), intitolata "un passo importante", C. Krause, il presidente della FLM, porta queste parole di Martino Lutero: "Non siamo noi che possiamo conservare la Chiesa. Non sono stati neppure i nostri predecessori. Non saranno neppure i nostri discendenti, ma è stato, è e sarà, unicamente colui che dice: Io sono con voi tutti i giorni fino alla fine del mondo." (Krause, “Un passo importante”, 713).
} 
Vorremo concludere finalmente dicendo che noi cristiani del XXI secolo abbiamo bisogno di un "nuovo ottimismo ecumenico" -secondo le parole del cardinale Kasper-, ovvero un nuovo slancio verso l'ecumenismo; poiché "l'unità della Chiesa è un dono dello Spirito di Dio, che ci è stato solennemente promesso". ${ }^{87}$

\section{Bibliografia}

Barth, Karl. Carta a los Romanos. Madrid: Biblioteca de Autores Cristianos, 1998 [2002].

Birmelé, André. “Uniti sulla giustificazione." Il Regno Documenti 45, 3/852 (2000): 127-136.

Canals Vidal, Francisco. En torno al diálogo católico-protestante. Barcelona: Herder, 1966.

Cassidy, Edward I. “Continuare la costruzione." Il Regno Documenti 44, 21/848 (1999): 712.

Cereti, Giovanni; Sever J. Voicu (a cura). Enchiridion oecumenicum. Vol. 1. Documenti del dialogo teologico interconfessionale. Dialoghi internazionali 1931-1984. Bologna: EDB, 1986.

Cereti, Giovanni (a cura). Enchiridion oecumenicum. Vol. 2. Documenti del dialogo teologico interconfessionale. Dialoghi locali 1965-1987. Bologna: EDB, 1989.

Cereti, Giovanni; J. F. Puglisi (a cura). Enchiridion oecumenicum. Vol. 3. Documenti del dialogo teologico interconfessionale. Dialoghi internazionali 1985-1994. Bologna: EDB, 1995.

Cereti, Giovanni; J. F. Puglisi, James (a cura). Enchiridion Oecumenicum. Vol. 7. Documenti del dialogo teologico interconfessionale. Dialoghi internazionali 19952005. Bologna: EDB, 2006.

Concilio di Trento. “Decreto sulla giustificazione (1547)." In Enchiridion symbolorum, definitionum et delcarationum de rebus fidei et morum, a cura di H. Denzinger e P. Hünermann, 647-671. Bologna: Edizioni Dehoniane, 2003.

Concilio Vaticano II. "Decreto sull'ecumenismo Unitatis redintegratio (1964)." Vatican, http://www.vatican.va/archive/hist_councils/ii_vatican_council/documents/ vat-ii_decree_19641121_unitatis-redintegratio_it.html (consultato 20 di aprile 2015).

${ }^{87}$ Idem, “La Dichiarazione congiunta sulla dottrina della giustificazione”, 14. 
De Papiol, Remigio. El protestantismo ante la Biblia. Barcelona: Eugenio Subirana, 1923.

Dettloff, W. "Justificación." En Conceptos fundamentales de la teología, editado por H. Fries, II, 480-490. Madrid: Cristiandad, 1966.

Eichholz, Georg. El Evangelio de Pablo. Esbozo de la teología paulina. Biblioteca de Estudios Bíblicos 17. Salamanca: Ediciones Sígueme, 1977.

Giovanni Paolo II. "Lettera enciclica Ut unum sint sull'impegno ecumenico (1995)." Vatican, http://w2.vatican.va/content/john-paul-ii/it/encyclicals/documents/ hf_jp-ii_enc_25051995_ut-unum-sint.html (consultato 20 di aprile 2015). . "Limpegno della chiesa nell'ecumenismo deriva da una decisione irrevocabile." In Insegnamenti di Giovanni Paolo II. Vol. VIII 1 (1985): 1988. Città del Vaticano: Liberia Editrice Vaticana, 1985.

- "Un ecumenismo promettente per il futuro può esistere solo ponendosi disinteressatamente di fronte alla verità.” In Insegnamenti di Giovanni Paolo II. Vol. XIX, 1 (1996), 1561-1567. Città del Vaticano: Liberia Editrice Vaticana, 1998.

Jeremias, Joachim. Abbá y el mensaje central del Nuevo Testamento. Biblioteca de Estudios Bíblicos 30. Salamanca: Ediciones Sígueme, 1981 [1989].

Jüngel, Eberhard. El Evangelio de la justificación del impio como centro de la fe cristiana. Estudio teológico en perspectiva ecuménica. Salamanca: Ediciones Sígueme, 2004.

Kasper, Walter. Cosechar los frutos. Aspectos básicos de la fe cristiana en el diálogo ecuménico. Santander: Sal Terrae, 2010.

- "La Dichiarazione congiunta sulla dottrina della giustificazione. Un motivo di speranza." Euntes Docete 53/1 (2000): 7-14.

. "Tutti siano una cosa sola." Il Regno Documenti 1 (2008): 1-5.

. "Vi raccoglierò, vi radunerò." Il Regno Documenti 44, 21/848 (1999): 710.

Krause, Christian. “Un passo importante.” Il Regno Documenti 44, 21/848 (1999): 713.

Küng, Hans. La justificación. Doctrina de Karl Barth y una interpretación católica. Barcelona: Editorial Estela, 1967.

Ladaria, Luis Fernando. Teología del pecado original y de la gracia. Antropología teológica especial. Madrid: Biblioteca de Autores Cristianos, 1993 [1997].

Lehmann, Karl. "Fondamentalmente uniti." Il Regno Documenti 44, 21/848 (1999): 708-712. 
Mondin, Batista. “Lutero Martin.” In Dizionario dei teologi, por B. Mondin, 357-370. Bologna: Edizioni Studio Domenicano, 1992.

Pattaro, G. "Ecumenismo." In Nuovo dizionario di teologia, a cura di G. Barbaglio e S. Dianich, 349-370. Cinisello Balsamo (MI): Edizioni Paoline, 1976 [1985].

Pontificio Consiglio per la Promozione dell'Unità dei Cristiani-Federazione Luterana Mondiale. "Dichiarazione congiunta sulla dottrina della giustificazione." In Enchiridion Oecumenicum: Documenti del dialogo teologico interconfessionale. Vol. 7. Documenti del dialogo teologico interconfessionale. Dialoghi internazionali 1995-2005, Giovanni Cereti, James F. Puglisi (a cura), 885-913. Bologna: EDB, 2006.

Pontificio Consiglio per l'Unità dei Cristiani. "Risposta sulla giustificazione." Il Regno Documenti 15 (1998): 497-499.

Ruiz de la Peña, Juan Luis. El don de Dios. Antropología especial. Colección Presencia teológica 63. Santander: Sal Terrae, 1991.

Semeraro, M. "Jüngel Eberhard." In Dizionario dei teologi dal primo secolo ad oggi, a cura di L. Pacomio e G. Occhipinti, 720-721, Casale Monferrato: Edizioni Piemme, 1998.

Track, Joachim. "Limportanza della Dichiarazione congiunta." Il Regno Documenti 44, 21/848 (1999): 713-715.

Wilckens Ulrich. La Carta a los Romanos. Rom 6-16. Tomo II. Biblioteca de Estudios Bíblicos 62. Salamanca: Ediciones Sígueme, 1989 [1992]. 
